\title{
Entropy-Based Proofs of Combinatorial Results on Bipartite Graphs
}

\author{
Igal Sason \\ Andrew and Erna Viterbi Faculty of Electrical and Computer Engineering \\ Technion-Israel Institute of Technology, Haifa 3200003, Israel \\ E-mail: sason@ee.technion.ac.il
}

\begin{abstract}
This work considers new entropy-based proofs of some known, or otherwise refined, combinatorial bounds for bipartite graphs. These include upper bounds on the number of the independent sets, lower bounds on the minimal number of colors in constrained edge coloring, and lower bounds on the number of walks of a given length in bipartite graphs. The proofs of these combinatorial results rely on basic properties of the Shannon entropy.
\end{abstract}

\section{INTRODUCTION}

The Shannon entropy serves as a powerful tool in various combinatorial and graph-theoretic applications (see, e.g., the tutorials in [8] and [20], as well as [5], [6], [17], [18], [19]).

Combinatorial properties of bipartite graphs are of great interest in graph theory, combinatorics, modern coding theory, and information theory. Entropy-based proofs pertain to the following aspects of such combinatorial properties:

1) Enumeration of the independent sets in graphs: Many important structures can be modeled by independent sets in a graph, i.e., subsets of vertices in a graph where none of them are connected by an edge. If a graph models some kind of incompatibility, then an independent set in this graph represents a mutually compatible collection. An application of Shearer's lemma to obtain a tight upper bound on the number of independent sets in $d$-regular bipartite graphs, and a recent extension of this informationtheoretic proof for irregular bipartite graphs (which is tight for bipartite graphs that are regular on one side) are available in [13] and [24], respectively. The informationtheoretic literature considers independent sets and their enumeration in [11], [13], [15], [16], and recently in [24].

2) Enumeration of perfect matchings in bipartite graphs: An elegant entropy-based proof of Bregman's theorem, which is a tight upper bound on the permanent of square matrices with binary entries, was introduced in [21]. The permanent of such a matrix is equivalent to the number of perfect matchings of the induced bipartite graph, so [21] provides an information-theoretic proof for a tight upper bound on the number of perfect matchings in bipartite graphs.

3) Moore's bound: The girth of a graph is the length of its shortest cycle, and it is of interest in graph theory. The girth is also of importance in the realm of modern coding theory with respect to codes defined on (bipartite) graphs and their iterative message-passing decoding algorithms (see, e.g., [7] and [22, Problems 3.25-3.37]). Moore's bound provides an upper bound on the girth of irregular graphs as a function of their number of vertices and their average degree [1]. An entropy-based proof of Moore's bound for graphs (and bipartite graphs) was introduced in [2].

4) Additional combinatorial properties of bipartite graphs: In [12], a new conditional entropy inequality was derived, followed by a study of two of its combinatorial applications to bipartite graphs. These include a derivation of a lower bound on the minimal number of colors in (rich) graph coloring, and a derivation of a lower bound on the biclique cover number of bipartite graphs.

5) Although not directly related to bipartite graphs, a variant of Shearer's lemma for the relative entropy was introduced in [16] (see Corollary 7 and Remark 9 there), and also independently (several years later) in [10]. Furthermore, the work in [10] applies this variant to obtain a Chernofftype bound for the sum of read- $k$ functions of independent variables (i.e., a set of functions where each variable participates in at most $k$ functions); it is then used in [10] to derive a probabilistic bound on the number of triangles in random graphs, constructed by the Erdôs-Rényi model.

There is a long history of applying entropy inequalities for obtaining combinatorial results, and the present paper aims to further develop this connection. It provides new entropybased proofs of known, or otherwise refined, combinatorial bounds for bipartite graphs. This paper has the following structure: Section $\amalg$ provides preliminaries and notation, and Sections [II V suggest entropy-based proofs of combinatorial properties of bipartite graphs. Specifically, Section III refers to a generalized information-theoretic approach for bounding the number of independent sets in bipartite graphs. The material in Section III] outlines our recent work in [24]. Sections IV] and V provide entropy-based proofs of two combinatorial results for bipartite graphs. Section IV] generalizes a conditionalentropy inequality in [12] (Proposition 2 here). In continuation to [12, Section IV], the generalized inequality is used to derive a lower bound on the minimal number of colors in constrained graph colorings of bipartite graphs. Section $\mathrm{V}$ provides entropy-based lower bounds on the number of walks of a given length in bipartite graphs (Proposition 3), relying on a work on the Moore bound [1], and its later informationtheoretic formulation in [2]. 


\section{Preliminaries And Notation}

Let $G$ be an undirected graph, and let $\mathrm{V}(G)$ and $\mathrm{E}(G)$ denote, respectively, the sets of vertices and edges in $G$.

A graph is called bipartite if it has two types of vertices, and an edge cannot connect vertices of the same type; we refer to the vertices of a bipartite graph $G$ as left and right vertices.

A graph $G$ is called complete if every vertex $v \in \mathrm{V}(G)$ is connected to all the other vertices in $\mathrm{V}(G) \backslash\{v\}$ (and not to itself); similarly, a bipartite graph is called complete if every vertex is connected to all the vertices of the other type in the graph. A complete $(d-1)$-regular graph is denoted by $K_{d}$, having a number of vertices $\left|\mathrm{V}\left(K_{d}\right)\right|=d$, and a number of edges $\left|\mathrm{E}\left(K_{d}\right)\right|=\frac{1}{2} d(d-1)$. Likewise, a complete $d$-regular bipartite graph is denoted by $K_{d, d}$, having a number of vertices $\left|\mathrm{V}\left(K_{d, d}\right)\right|=2 d$ (i.e., $d$ vertices of each of the two types), and a number of edges $\left|\mathrm{E}\left(K_{d, d}\right)\right|=d^{2}$.

An independent set of an undirected graph $G$ is a subset of its vertices such that none of the vertices in this subset are adjacent (i.e., none of them are joined by an edge). Let $\mathcal{I}(G)$ denote the set of all the independent sets in $G$, and let $|\mathcal{I}(G)|$ denote the number of independent sets in $G$.

The tensor product $G \times H$ of two graphs $G$ and $H$ is a graph such that the vertex set of $G \times H$ is the Cartesian product $\mathrm{V}(G) \times \mathrm{V}(H)$, and two vertices $(g, h),\left(g^{\prime}, h^{\prime}\right) \in \mathrm{V}(G \times H)$ are adjacent if and only if $g$ is adjacent to $g^{\prime}$, and $h$ is adjacent to $h^{\prime}$ (i.e., $\left(g, g^{\prime}\right) \in \mathrm{E}(G)$ and $\left.\left(h, h^{\prime}\right) \in \mathrm{E}(H)\right)$.

By the definition of a complete $d$-regular graph $K_{d}$, the graph $K_{2}$ is specialized to two vertices that are connected by an edge. Let us label the two vertices in $K_{2}$ by 0 and 1 . For a graph $G$, the tensor product $G \times K_{2}$ is a bipartite graph, called the bipartite double cover of $G$, where the set of vertices in $G \times K_{2}$ is given by

$$
\mathrm{V}\left(G \times K_{2}\right)=\{(v, i): v \in \mathrm{V}(G), i \in\{0,1\}\},
$$

and its set of edges is given by

$$
\mathrm{E}\left(G \times K_{2}\right)=\{((u, 0),(v, 1)):(u, v) \in \mathrm{E}(G)\} .
$$

Every edge $e=(u, v) \in \mathrm{E}(G)$ is mapped into the two edges $((u, 0),(v, 1)) \in \mathrm{E}\left(G \times K_{2}\right)$ and $((v, 0),(u, 1)) \in \mathrm{E}\left(G \times K_{2}\right)$ (since the graph $G$ is undirected). This implies that the numbers of vertices and edges in $G \times K_{2}$ are doubled in comparison to their respective numbers in $G$; moreover, every edge in $G$, which connects a pair of vertices of specified degrees, is mapped into two edges in $G \times K_{2}$ where each of these two edges connects a pair of vertices of the same specified degrees.

An edge coloring of a graph is an assignment of colors to its edges such that each two edges sharing a vertex in this graph have different colors. Finding the minimal number of colors in an edge coloring of a given graph is a classical problem in graph theory (see, e.g., [4, Chapter 5]).

The following further notation and basic facts are used:

- $\mathbb{N} \triangleq\{1,2, \ldots\}$ denotes the set of natural numbers.

- $X^{n} \triangleq\left(X_{1}, \ldots X_{n}\right)$ denotes an $n$-dimensional random vector of discrete random variables, having a joint probability mass function (PMF) that is denoted by $\mathrm{P}_{X^{n}}$.
- For every $n \in \mathbb{N}$, let $[1: n] \triangleq\{1, \ldots, n\}$;

- $X_{\mathcal{S}} \triangleq\left(X_{i}\right)_{i \in \mathcal{S}}$ is a random vector for an arbitrary nonempty subset $\mathcal{S} \subseteq[1: n]$; if $\mathcal{S}=\emptyset$, then conditioning on $X_{\mathcal{S}}$ is void. By continuous extension, the convention $0 \log 0=0$ is used.

Shearer's lemma extends the subadditivity property of the Shannon entropy.

Proposition 1 (Shearer's Lemma, [3]): Let $X_{1}, \ldots, X_{n}$ be discrete random variables, and let $\mathcal{S}_{1}, \ldots, \mathcal{S}_{m} \subseteq[1: n]$ include every element $i \in[1: n]$ in at least $k \geq 1$ of these subsets. Then,

$$
k \mathrm{H}\left(X^{n}\right) \leq \sum_{j=1}^{m} \mathrm{H}\left(X_{\mathcal{S}_{j}}\right) .
$$

Remark 1 ([24]): Inequality (3) holds even if the sets $\mathcal{S}_{1}, \ldots, \mathcal{S}_{m}$ are not necessarily included in $[1: n]$. To verify

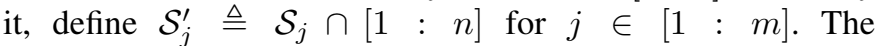
subsets $\mathcal{S}_{1}^{\prime}, \ldots, \mathcal{S}_{m}^{\prime}$ are included in $[1: n]$, and every element $i \in[1: n]$ continues to be included in at least $k \geq 1$ of these subsets. Hence, Proposition 1 can be applied to the subsets $\mathcal{S}_{1}^{\prime}, \ldots, \mathcal{S}_{m}^{\prime}$. By the monotonicity property of the entropy, the inclusion $\mathcal{S}_{j}^{\prime} \subseteq \mathcal{S}_{j}$ implies that $\mathrm{H}\left(X_{\mathcal{S}_{j}^{\prime}}\right) \leq \mathrm{H}\left(X_{\mathcal{S}_{j}}\right)$ for all $j \in[1: m]$, which then yields the satisfiability of (3).

\section{NuMBER OF INDEPENDENT SETS}

\section{A. Background}

This present section is focused on the problem of upper bounding the number of independent sets in a graph, expressed in terms of its degree distribution. For bipartite regular graphs, Kahn [13] established a tight upper bound using an information-theoretic approach, which is given as follows.

Theorem 1 (Kahn 2001, [13]): If $G$ is a bipartite $d$-regular graph with $n$ vertices, then

$$
|\mathcal{I}(G)| \leq\left(2^{d+1}-1\right)^{\frac{n}{2 d}} .
$$

Moreover, if $n$ is an even multiple of $d$, then the upper bound in the right side of (4) is tight, and it is obtained by a disjoint union of $\frac{n}{2 d}$ complete $d$-regular bipartite graphs $\left(K_{d, d}\right)$.

Kahn also conjectured in [13] an upper bound for general graphs. His conjectured bound was recently proved (after two decades) by Sah et al. (2019), using different techniques not involving information theory. Their tight bound is as follows:

Theorem 2 (Sah et al. 2019, [23]): Let $G$ be an undirected graph without isolated vertices or multiple edges connecting any pair of vertices. Let $\mathrm{d}_{v}$ denote the degree of a vertex $v \in \mathrm{V}(G)$. Then,

$$
|\mathcal{I}(G)| \leq \prod_{(u, v) \in \mathrm{E}(G)}\left(2^{\mathrm{d}_{u}}+2^{\mathrm{d}_{v}}-1\right)^{\frac{1}{\mathrm{~d}_{u} \mathrm{~d} v}}
$$

with equality if $G$ is a disjoint union of complete bipartite graphs.

The main contribution of our recent work in [24] is the extension of Kahn's information-theoretic proof technique to handle irregular bipartite graphs. In particular, when the bipartite graph is regular on one side, but it may be irregular 
in the other, the extended entropy-based proof technique yields the same bound that was conjectured by Kahn [13] and proved by Sah et al. [23].

The following result by Zhao [26] upper bounds the square of the number of independent sets of an arbitrary finite graph $G$ by the number of independent sets of the bipartite double cover of this graph (i.e., the tensor product of $G$ with $K_{2}$ ).

Theorem 3 (Zhao 2010, [26]): For every finite graph $G$ :

$$
|\mathcal{I}(G)|^{2} \leq\left|\mathcal{I}\left(G \times K_{2}\right)\right|
$$

As an application of Theorem 3, the extension of (5) from bipartite graphs to general graphs (without isolated vertices or multiple edges) was enabled in [9, Lemma 3] by relying on (6). Recall that every edge $e=(u, v) \in \mathrm{E}(G)$, which is connected in a graph $G$ to a pair of vertices of degrees $\mathrm{d}_{u}$ and $\mathrm{d}_{v}$, is mapped into two edges in the bipartite graph $G \times K_{2}$ where each one of these edges is connected to a pair of vertices of degrees $\mathrm{d}_{u}$ and $\mathrm{d}_{v}$ (see Section $(\Pi$ ). By inequality (6), and in view of the above proof for the setting of bipartite graphs, we obtain that for a general graph $G$

$$
\begin{aligned}
|\mathcal{I}(G)|^{2} & \leq\left|\mathcal{I}\left(G \times K_{2}\right)\right| \\
& \leq \prod_{\left(u^{\prime}, v^{\prime}\right) \in \mathrm{E}\left(G \times K_{2}\right)}\left(2^{\mathrm{d}_{u^{\prime}}}+2^{\mathrm{d}_{v^{\prime}}}-1\right)^{\frac{1}{\mathrm{~d}_{u^{\prime}{ }^{\mathrm{d}} v^{\prime}}}} \\
& =\prod_{(u, v) \in \mathrm{E}(G)}\left(2^{\mathrm{d}_{u}}+2^{\mathrm{d}_{v}}-1\right)^{\frac{2}{\mathrm{~d}^{\mathrm{d}} \mathrm{d} v}} .
\end{aligned}
$$

Finally, taking the square-roots of the left side of (7) and the right side of (9) gives (5) for general graphs.

In view of the above paragraph, it is sufficient to prove Theorem 2 for general graphs by confirming it in the special setting of bipartite graphs. The work in [23] recently proved Theorem 2 for bipartite graphs by using an induction on the number of vertices in a graph $G$, and by obtaining a recurrence inequality whose derivation involves judicious applications of Hölder's inequality (see [23, Sections 2 and 4]). The proof there does not rely on information theory.

\section{B. Contribution}

In a very recent paper [24], we provide an extension of the entropy-based proof by Kahn [13] from bipartite $d$-regular graphs to general bipartite graphs, and then we prove (5) for the family of bipartite graphs that are regular on one side (see [24, Section 4]). The proof in [24] follows the same recipe of Kahn's proof in [13] with some complications that arise from the non-regularity of the bipartite graphs. The proof in [24] deviates from the proof in [13] already at its starting point, by a proper adaptation of the proof technique to the general setting of irregular bipartite graphs, followed by a bit more complicated usage of Shearer's lemma (in light of Remark 10 and a more involved analysis. The reader is referred to the proof in [24, Section 4].

In [24, Section 5], we suggest a variant of the proof of Zhao's Inequality in (6) (given implicitly in [26, Lemma 2.1], and explicitly in a follow-up work by Galvin and Zhao [9]).
This forms in essence a reformulation of Zhao's proof, which is provided as follows.

Proof: Let $G$ be a finite graph, and let $|\mathrm{V}(G)|=n$. Label the vertices in the left and right sides of the bipartite graph $G \times K_{2}$ (i.e., the bipartite double cover of $G$ ) by $\{(i, 0)\}_{i=1}^{n}$ and $\{(i, 1)\}_{i=1}^{n}$, respectively.

Choose independently and uniformly at random two independent sets $\mathcal{S}_{0}, \mathcal{S}_{1} \in \mathcal{I}(G)$. For $i \in[1: n]$, let $X_{i}, Y_{i} \in\{0,1\}$ be random variables defined as $X_{i}=1$ if and only if $i \in \mathcal{S}_{0}$, and $Y_{i}=1$ if and only if $i \in \mathcal{S}_{1}$. Then, by the statistical independence and equiprobable selection of the two independent sets from $\mathcal{I}(G)$, we have

$$
\begin{aligned}
\mathrm{H}\left(X^{n}, Y^{n}\right) & =\mathrm{H}\left(X^{n}\right)+\mathrm{H}\left(Y^{n}\right) \\
& =2 \log |\mathcal{I}(G)|,
\end{aligned}
$$

where 10 holds since $X^{n}=\left(X_{1}, \ldots, X_{n}\right)$ and $Y^{n}=$ $\left(Y_{1}, \ldots, Y_{n}\right)$ are statistically independent (by construction), and (11) holds since they both have an equiprobable distribution over a set whose cardinality is $|\mathcal{I}(G)|$.

Consider the following set of vertices in $G \times K_{2}$ :

$$
\begin{aligned}
\mathcal{S} & \triangleq\left\{\mathcal{S}_{0} \times\{0\}\right\} \bigcup\left\{\mathcal{S}_{1} \times\{1\}\right\} \\
& =\bigcup_{i \in \mathcal{S}_{0}, j \in \mathcal{S}_{1}}\{(i, 0),(j, 1)\} .
\end{aligned}
$$

The set $\mathcal{S}$ is not necessarily an independent set in $G \times K_{2}$ since $((i, 0),(j, 1)) \in \mathrm{E}\left(G \times K_{2}\right)$ for all $i \in \mathcal{S}_{0}$ and $j \in \mathcal{S}_{1}$ for which $(i, j) \in \mathrm{E}(G)$ (see (2)). We next consider all $(i, j) \in$ $\mathrm{E}(G)$ such that $X_{i}=Y_{j}=1$. To that end, fix an ordering of all the $2^{n}$ subsets of $\mathrm{V}(G)$, and let $\mathcal{T} \in \mathrm{V}(G)$ be the first subset in this particular ordering that includes exactly one endpoint of each edge $(i, j) \in \mathrm{E}(G)$ for which $X_{i}=Y_{j}=1$. Consider the following replacements:

- If $(i, 0) \in \mathcal{S}$ and $i \in \mathcal{T}$, then $(i, 0)$ is replaced by $(i, 1)$;

- Likewise, if $(j, 1) \in \mathcal{S}$ and $j \in \mathcal{T}$, then $(j, 1)$ is replaced by $(j, 0)$.

Let $\widetilde{\mathcal{S}}$ be the set of new vertices after these possible replacements. Then, $\widetilde{S} \in \mathcal{I}\left(G \times K_{2}\right)$ since all adjacent vertices in $\mathcal{S}$ are no longer connected in $\widetilde{\mathcal{S}}$. Indeed, there is no way that after (say) a vertex $(i, 0)$ is replaced by $(i, 1)$, there is another replacement of a vertex $(j, 1)$ by $(j, 0)$, for some $j$ such that $(i, j) \in \mathrm{E}(G)$; otherwise, that would mean that $\mathcal{T}$ contains both $i$ and $j$, which is impossible by construction.

Similarly to the way $X^{n}, Y^{n} \in\{0,1\}^{n}$ were defined, let $\widetilde{X}^{n}, \widetilde{Y}^{n} \in\{0,1\}^{n}$ be defined such that, for all $i \in[1: n]$, $\widetilde{X}_{i}=1$ if and only if $(i, 0) \in \widetilde{\mathcal{S}}$, and $\widetilde{Y}_{i}=1$ if and only if $(i, 1) \in \widetilde{\mathcal{S}}$.

The mapping from $\left(X^{n}, Y^{n}\right)$ to $\left(\tilde{X}^{n}, \tilde{Y}^{n}\right)$ is injective. Indeed, it is shown to be injective by finding all indices $(i, j) \in \mathrm{E}(G)$ such that $\widetilde{X}_{i}=\widetilde{X}_{j}=1$ or $\widetilde{Y}_{i}=\widetilde{Y}_{j}=1$, finding the first subset $\mathcal{T} \in \mathrm{V}(G)$ according to our previous fixed ordering of the $2^{n}$ subsets of $\mathrm{V}(G)$ that includes exactly one endpoint of each such edge $(i, j) \in \mathrm{E}(G)$, and performing the reverse operation to return back to $X^{n}$ and $Y^{n}$ (e.g., if $(i, j) \in \mathrm{E}(G), \tilde{X}_{i}=\tilde{X}_{j}=1$ and $i \in \mathcal{T}$ while $j \notin \mathcal{T}$, 
then $\widetilde{X}_{i}=1$ is transformed back to $Y_{i}=1$, and $\widetilde{X}_{j}=1$ is transformed back to $X_{j}=1$ ). Consequently, we get

$$
\begin{aligned}
\mathrm{H}\left(X^{n}, Y^{n}\right) & =\mathrm{H}\left(\widetilde{X}^{n}, \widetilde{Y}^{n}\right) \\
& \leq \log \left|\mathcal{I}\left(G \times K_{2}\right)\right|,
\end{aligned}
$$

where (14) holds by the injectivity of the mapping from $\left(X^{n}, Y^{n}\right)$ to $\left(\widetilde{X}^{n}, \widetilde{Y}^{n}\right)$, and (15) holds since $\widetilde{S}$ is an independent set in $G \times K_{2}$, which implies that $\left(\widetilde{X}^{n}, \widetilde{Y}^{n}\right)$ can get at most $\left|\mathcal{I}\left(G \times K_{2}\right)\right|$ possible values (by definition, there is a oneto-one correspondence between $\widetilde{\mathcal{S}}$ and $\left.\left(\widetilde{X}^{n}, \widetilde{Y}^{n}\right)\right)$. Combining (10), (11), (14) and (15) gives

$$
2 \log |\mathcal{I}(G)| \leq \log \left|\mathcal{I}\left(G \times K_{2}\right)\right|,
$$

which gives (6) by exponentiation of both sides of (16).

\section{Outlook}

In [11, Corollary 6.2] and its related discussion, Johnson, Kontoyiannis and Madiman provided an upper bound on the entropy of the size of a random independent set in a claw-free graph (i.e., a graph that does not contain the complete bipartite graph $K_{1,3}$ as an induced subgraph). In light of a connection between the size of a random independent set and the total number of independent sets (the latter is the partition function of the hard-core model with fugacity 1 , see the definition of the independence polynomial in [26] for details), it is left for future work to study if the results in [24] can be applied to yield bounds on the size of a random independent set, or bounds on the partition function with a general fugacity.

\section{Edge Coloring of Bipartite Graphs}

In [12, Theorem 1], the following result is proved.

Theorem 4 (Kaced et al. 2018, [12]): Let $A, X$ and $Y$ be discrete random variables taking their values in the sets $\mathcal{A}, \mathcal{X}, \mathcal{Y}$, respectively, with a joint probability mass function $\mathrm{P}_{A, X, Y}$. If for every $(x, y) \in \mathcal{X} \times \mathcal{Y}$, there exists at most one element $a \in \mathcal{A}$ such that $\mathrm{P}_{A, X}(a, x) \mathrm{P}_{A, Y}(a, y)>0$ then

$$
\mathrm{H}(A \mid X)+\mathrm{H}(A \mid Y) \leq \mathrm{H}(A) .
$$

We next provide a modest generalization of Theorem 4 which suggests an extension of the result in [12, Corollary 1] with respect to edge coloring of bipartite graphs.

Proposition 2: Let $A, X, Y$ be discrete random variables taking values in sets $\mathcal{A}, \mathcal{X}, \mathcal{Y}$, respectively. Then,

$$
\mathrm{H}(A \mid X)+\mathrm{H}(A \mid Y) \leq \mathrm{H}(A)+\log m,
$$

where

$$
m \triangleq \sup _{(x, y) \in \mathcal{X} \times \mathcal{Y}}\left|\left\{a \in \mathcal{A}: \mathrm{P}_{A, X}(a, x) \mathrm{P}_{A, Y}(a, y)>0\right\}\right| .
$$

Proof: Consider the PMF

$$
\mathrm{P}_{A, X, Y}^{\prime}(a, x, y) \triangleq \begin{cases}\frac{\mathrm{P}_{A, X}(a, x) \mathrm{P}_{A, Y}(a, y)}{\mathrm{P}_{A}(a)}, & \mathrm{P}_{A}(a)>0 \\ 0, & \text { otherwise, }\end{cases}
$$

which refers to the case where $A$ is chosen according to the PMF $\mathrm{P}_{A}$, and $X$ and $Y$ are conditionally independent given $A$ with the conditional PMFs $\mathrm{P}_{X \mid A}$ and $\mathrm{P}_{Y \mid A}$, respectively.

$$
\begin{aligned}
\mathrm{H} & (A \mid X)+\mathrm{H}(A \mid Y)-\mathrm{H}(A) \\
& =\mathrm{H}(A, X)+\mathrm{H}(A, Y)-\mathrm{H}(X)-\mathrm{H}(Y)-\mathrm{H}(A) \\
& =\sum_{a, x, y} \mathrm{P}_{A, X, Y}(a, x, y) \log \left(\frac{\mathrm{P}_{X}(x) \mathrm{P}_{Y}(y) \mathrm{P}_{A}(a)}{\mathrm{P}_{A, X}(a, x) \mathrm{P}_{A, Y}(a, y)}\right) \\
& =\sum_{a, x, y} \mathrm{P}_{A, X, Y}^{\prime}(a, x, y) \log \left(\frac{\mathrm{P}_{X}(x) \mathrm{P}_{Y}(y) \mathrm{P}_{A}(a)}{\mathrm{P}_{A, X}(a, x) \mathrm{P}_{A, Y}(a, y)}\right)
\end{aligned}
$$

where the last equality holds since the following equalities are satisfied by the marginals of $\mathrm{P}_{A, X, Y}$ and $\mathrm{P}_{A, X, Y}$ :

$$
\mathrm{P}_{A, X}=\mathrm{P}_{A, X}^{\prime}, \quad \mathrm{P}_{A, Y}=\mathrm{P}_{A, Y}^{\prime},
$$

and the terms $\mathrm{H}(A, X), \mathrm{H}(A, Y), \mathrm{H}(X), \mathrm{H}(Y), \mathrm{H}(A)$ in the right-side of (20) only depend on the marginal PMFs that appear in 211. Due to the concavity of the logarithmic function, invoking Jensen's inequality gives

$$
\begin{aligned}
& \mathrm{H}(A \mid X)+\mathrm{H}(A \mid Y)-\mathrm{H}(A) \\
& \leq \log \sum_{(a, x, y) \in \operatorname{supp}\left(\mathrm{P}^{\prime}\right)} \frac{\mathrm{P}^{\prime}{ }_{A, X, Y}(a, x, y) \mathrm{P}_{X}(x) \mathrm{P}_{Y}(y) \mathrm{P}_{A}(a)}{\mathrm{P}_{A, X}(a, x) \mathrm{P}_{A, Y}(a, y)} \\
& =\log \left(\sum_{(a, x, y): \mathrm{P}^{\prime}} \sum_{A, X, Y} \mathrm{P}_{X}(x) \mathrm{P}_{Y}(y)\right)
\end{aligned}
$$

For all $(x, y) \in \mathcal{X} \times \mathcal{Y}$, let

$$
\begin{aligned}
m(x, y) & \triangleq\left|\left\{a \in \mathcal{A}: \mathrm{P}_{A, X}(a, x) \mathrm{P}_{A, Y}(a, y)>0\right\}\right| \\
& =\left|\left\{a \in \mathcal{A}: \mathrm{P}_{A, X, Y}^{\prime}(a, x, y)>0\right\}\right| .
\end{aligned}
$$

Then,

$$
\begin{gathered}
\sum_{(a, x, y): \mathrm{P}^{\prime}{ }_{A, X, Y}(a, x, y)>0} \mathrm{P}_{X}(x) \mathrm{P}_{Y}(y) \\
=\sum_{x, y}\left\{m(x, y) \mathrm{P}_{X}(x) \mathrm{P}_{Y}(y)\right\} \\
\leq m \sum_{x, y}\left\{\mathrm{P}_{X}(x) \mathrm{P}_{Y}(y)\right\} \\
=m,
\end{gathered}
$$

where (25) holds by (23), and (26) holds by (19). Finally, combining (22) and (25)-(27) gives (18).

Proposition 2 is useful if $\log m<\mathrm{H}(A)$, and otherwise (18) becomes trivial.

In view of Proposition 2 and the proof of [12, Corollary 1], the following result follows readily.

Corollary 1: Consider a bipartite graph $G$ with minimal left and right degrees that are equal to $d_{\mathrm{L}}$ and $d_{\mathrm{R}}$, respectively. Consider an edge coloring of $G$ where, in addition to the requirement that every two edges sharing a node have different colors, it is satisfied that for all pairs of left node $v_{\mathrm{L}}$ and right node $v_{\mathrm{R}}$ in $\mathrm{V}(G)$, there are at most $m$ colors touching both 
$v_{\mathrm{L}}$ and $v_{\mathrm{R}}$. Then, the number of colors in every such edge coloring of $G$ is at least $\frac{d_{\mathrm{L}} d_{\mathrm{R}}}{m}$.

Proof: It is similar to the proof of [12, Corollary 1], with the only modification of using (18) with an arbitrary $m \in \mathbb{N}$ (instead of (17), referring the special case where $m=1$ ).

By Vizing's theorem on edge coloring of graphs [25] (see [4. Theorem 5.3.2]), the number of colors needed to edge color a simple graph (i.e., an undirected graph containing no graph loops or multiple edges) is either equal to its maximal degree $d_{\max }$ or to $d_{\max }+1$. Furthermore, for bipartite graphs, the number of colors is always equal to $d_{\max }$.

The following simple consequence of Corollary 1 motivates the extension of the result in [12, Corollary 1] for constrained edge colorings of bipartite graphs. It provides a refinement of Vizing's theorem for regular bipartite graphs.

Corollary 2: If $G$ is a $d$-regular bipartite graph, and there is a requirement on the richness of the colors in the sense that at most $m<d$ colors touch every pair of left and right vertices in $G$, then the number of required colors is at least $\frac{d^{2}}{m}$ (which is strictly larger than $d_{\max }=d$ ).

\section{Number of Walks of a Given Length}

The present section derives lower bounds on the number of walks of a given length in bipartite graphs, based on basic properties of the Shannon entropy. These results rely on the work by Alon, Hoory and Linial on the Moore bound [1], and its later information-theoretic formulation due to Babu and Radhakrishnan [2].

We introduce here the refined bounds in (30) and (32), which are expressed in terms of Shannon entropies of probability mass functions that are induced by the degree distributions of the bipartite graph; these lower bounds tighten the bounds in (31) and (33), respectively.

Proposition 3: Let $G$ be a bipartite graph with a disjoint partition of its vertex set $\mathrm{V}(G)$ to sets of left and right vertices $\mathcal{U}$ and $\mathcal{V}$, respectively, with $|\mathcal{U}|=m$ and $|\mathcal{V}|=n$. Let $\mathcal{P}_{k}$ be the set of all walks of a given length $k \in \mathbb{N}$, where edges may be repeated. Let $\mathrm{d}_{r}$ denote the degree of a vertex $r \in \mathrm{V}(G)$, and let $P$ and $Q$ be PMFs defined, respectively, on $\mathcal{U}$ and $\mathcal{V}$ as follows:

$$
\begin{aligned}
& \mathrm{P}(u) \triangleq \frac{\mathrm{d}_{u}}{|\mathrm{E}(G)|}, \quad u \in \mathcal{U}, \\
& \mathrm{Q}(v) \triangleq \frac{\mathrm{d}_{v}}{|\mathrm{E}(G)|}, \quad v \in \mathcal{V} .
\end{aligned}
$$

1) If $k$ is odd, then

$$
\begin{aligned}
\left|\mathcal{P}_{k}\right| & \geq|\mathrm{E}(G)|^{k} \exp \left(-\frac{1}{2}(k-1)[\mathrm{H}(P)+\mathrm{H}(Q)]\right) \\
& \geq \frac{|\mathrm{E}(G)|^{k}}{(m n)^{\frac{k-1}{2}}} .
\end{aligned}
$$

2) If $k$ is even, then

$$
\begin{aligned}
\left|\mathcal{P}_{k}\right| \geq & |\mathrm{E}(G)|^{k} \exp \left(-\left(\frac{1}{2} k-1\right)[\mathrm{H}(P)+\mathrm{H}(Q)]\right) \\
& \cdot \exp (-\min \{\mathrm{H}(P), \mathrm{H}(Q)\}) \\
\geq & \frac{|\mathrm{E}(G)|^{k}}{(m n)^{\frac{k}{2}-1} \min \{m, n\}}
\end{aligned}
$$

with equalities in (30)-33) if the bipartite graph $G$ is regular.

Proof: We prove Proposition 3 when $k \in \mathbb{N}$ is odd. The proof when $k$ is even is essentially similar.

Let $k \triangleq 2 \ell+1$ with $\ell \in \mathbb{N}$, and select a random walk of length $k$ in the bipartite graph $G$ by the following procedure:

1) The edge $E_{\ell+1} \in \mathrm{E}(G)$ is selected uniformly at random among all edges in $G$. Let $E_{\ell+1}=\left(U_{\ell+1}, V_{\ell+1}\right)$ with $U_{\ell+1}$ and $V_{\ell+1}$ denoting, respectively, the left and right vertices attached to this edge;

2) Given $E_{\ell+1}$, the edge $E_{\ell}=\left(U_{\ell}, V_{\ell}\right) \in \mathrm{E}(G)$ is selected uniformly at random among all edges in $G$ with an endpoint at the given vertex $U_{\ell+1}$ (so $\left.U_{\ell+1}=V_{\ell}\right)$;

3) Similarly, given $E_{\ell+1}$, the edge $E_{\ell+2}$ is selected uniformly at random, independently of $E_{\ell}$, among all edges in $G$ with an endpoint at the given vertex $V_{\ell+1}$ (so, $\left.U_{\ell+2}=V_{\ell+1}\right)$;

4) Given $E_{\ell}$, the edge $E_{\ell-1}$ is selected uniformly at random among all edges in $G$ with an endpoint that coincides with the already selected endpoint $U_{\ell}$ of $E_{\ell}$ (see Step 2), and it is conditionally independent of the edges $E_{\ell+2}$ and $E_{\ell+1}$;

5) Given $E_{\ell+2}$, the edge $E_{\ell+3}$ is selected uniformly at random among all edges in $G$ with an endpoint that coincides with the already selected endpoint $V_{\ell+2}$ of $E_{\ell+2}$ (see Step 3), and it is conditionally independent of $E_{\ell+1}, E_{\ell}$ and $E_{\ell-1}$;

6) The process of selecting the edges of the walk is continued this way, and the following Markov chain is constructed:

$$
E_{1} \multimap \ldots \multimap E_{\ell} \multimap-E_{\ell+1} \multimap E_{\ell+2} \ldots \multimap-E_{2 \ell+1} .
$$

By the construction of a $k$-length random walk in $G$ as above,

$$
\begin{aligned}
\log \left|\mathcal{P}_{k}\right| \geq & \mathrm{H}\left(E_{1}, \ldots, E_{2 \ell+1}\right) \\
= & \mathrm{H}\left(E_{\ell+1}\right)+\left[\mathrm{H}\left(E_{\ell+2} \mid E_{\ell+1}\right)+\mathrm{H}\left(E_{\ell} \mid E_{\ell+1}\right)\right] \\
& +\left[\mathrm{H}\left(E_{\ell+3} \mid E_{\ell+2}\right)+\mathrm{H}\left(E_{\ell-1} \mid E_{\ell}\right)\right]+\ldots \\
& +\left[\mathrm{H}\left(E_{2 \ell+1} \mid E_{2 \ell}\right)+\mathrm{H}\left(E_{1} \mid E_{2}\right)\right]
\end{aligned}
$$

where (35) holds since $\left(E_{1}, \ldots, E_{2 \ell+1}\right)$ is a random walk in $\mathcal{P}_{k}$; (36) holds by the chain rule of the Shannon entropy and the Markovity property in (34). By Step 1 of the construction,

$$
\mathrm{H}\left(E_{\ell+1}\right)=\log |\mathrm{E}(G)|
$$

and, by Steps 2 and 3 of the construction,

$$
\begin{aligned}
& \mathrm{H}\left(E_{\ell+2} \mid E_{\ell+1}\right)=\sum_{v \in V}\left\{\frac{\mathrm{d}_{v}}{|\mathrm{E}(G)|} \cdot \log \mathrm{d}_{v}\right\}, \\
& \mathrm{H}\left(E_{\ell} \mid E_{\ell+1}\right)=\sum_{u \in U}\left\{\frac{\mathrm{d}_{u}}{|\mathrm{E}(G)|} \cdot \log \mathrm{d}_{u}\right\} .
\end{aligned}
$$


Moreover, by Steps $4-6$, for all $j \in\{1, \ldots, \ell\}$,

$$
\begin{aligned}
& \mathrm{H}\left(E_{\ell+j+1} \mid E_{\ell+j}\right)=\mathrm{H}\left(E_{\ell+2} \mid E_{\ell+1}\right), \\
& \mathrm{H}\left(E_{\ell+1-j} \mid E_{\ell+2-j}\right)=\mathrm{H}\left(E_{\ell} \mid E_{\ell+1}\right) .
\end{aligned}
$$

For $k=2 \ell+1$ with $\ell \in \mathbb{N}$, it follows that

$$
\begin{aligned}
& \log \left|\mathcal{P}_{k}\right| \geq \log |\mathrm{E}(G)|+ {\left[\sum_{u \in U}\left\{\frac{\mathrm{d}_{u}}{|\mathrm{E}(G)|} \cdot \log \mathrm{d}_{u}\right\}\right.} \\
&\left.+\sum_{v \in V}\left\{\frac{\mathrm{d}_{v}}{|\mathrm{E}(G)|} \cdot \log \mathrm{d}_{v}\right\}\right] \\
&=\log |\mathrm{E}(G)|+\ell {\left[\sum_{u \in U}\left\{\frac{\mathrm{d}_{u}}{|\mathrm{E}(G)|} \cdot \log \frac{\mathrm{d}_{u}}{|\mathrm{E}(G)|}\right\}\right.} \\
&+ \sum_{v \in V}\left\{\frac{\mathrm{d}_{v}}{|\mathrm{E}(G)|} \cdot \log \frac{\mathrm{d}_{v}}{|\mathrm{E}(G)|}\right\} \\
&+2 \log |\mathrm{E}(G)|] \\
&=(2 \ell+1) \log |\mathrm{E}(G)|-\ell[\mathrm{H}(P)+\mathrm{H}(Q)] \\
&=k \log |\mathrm{E}(G)|- \frac{1}{2}(k-1)[\mathrm{H}(P)+\mathrm{H}(Q)],
\end{aligned}
$$

where (42) holds by (35)-(41); (43) holds since ( $G$ is bipartite)

$$
\sum_{u \in U} \mathrm{~d}_{u}=|\mathrm{E}(G)|=\sum_{v \in V} \mathrm{~d}_{v} ;
$$

(44) holds by the definition of the PMFs $P$ and Q in (28) and (29), respectively. Finally, exponentiating the left side in (43) and the right side in (45) gives the lower bound in (30).

The transition from the lower bound on $\left|\mathcal{P}_{k}\right|$ in the right side of (30) to the looser lower bound in the right side of (31) holds since $|\mathcal{U}|=m$ and $|\mathcal{V}|=n$ yields (see (28) and (29))

$$
\mathrm{H}(P) \leq \log m, \quad \mathrm{H}(Q) \leq \log n .
$$

We finally show that the lower bound in the right side of (31) is achieved if $G$ is a regular bipartite graph. Let $G$ be a bipartite graph with fixed degrees $d_{\mathrm{L}}$ and $d_{\mathrm{R}}$ on its left and right sides, respectively. Then, $m d_{\mathrm{L}}=|\mathrm{E}(G)|=n d_{\mathrm{R}}$, and

$$
\frac{|\mathrm{E}(G)|^{k}}{(m n)^{\frac{k-1}{2}}}=|\mathrm{E}(G)|\left(d_{\mathrm{L}} d_{\mathrm{R}}\right)^{\frac{k-1}{2}},
$$

which is the number of $k$-length walks in $G$ for odd $k$.

A certain non-returning walk was considered in [1] for graphs of minimum degree at least 2 . It is left for a future study to examine the suitability of the same idea to yield bounds similar to Proposition 3 on the number of $k$-length trails (i.e., walks with no repeated edges), and the number of $k$-length paths (i.e., walks without repeated edges and vertices).

\section{ACKNOWLEDGEMENT}

Constructive comments by the three anonymous reviewers are gratefully acknowledged.

\section{REFERENCES}

[1] N. Alon, S. Hoory and N. Linial, "The Moore bound for irregular graphs," Graphs and Combinatorics, vol. 18, no. 1, pp. 53-57, 2002.

[2] S. A. Babu and J. Radhakrishnan, "An entropy-based proof for the Moore bound for irregular graphs," Perspectives on Computational Complexity, pp. 173-182, (Editors: M. Agrawal and V. Arvind), Birkhäuser, 2014.

[3] F. R. K. Chung, L. R. Graham, P. Frankl and J. B. Shearer, "Some intersection theorems for ordered sets and graphs," Journal of Combinatorial Theory, Series A, vol. 43, no. 1, pp. 23-37, 1986.

[4] R. Diestel, Graph Theory, Fifth Edition, Springer, 2017.

[5] P. Erdôs and A. Rényi, "On two problems of information theory," Publications of the Mathematical Institute of the Hungarian Academy of Sciences, vol. 8, pp. 241-254, 1963.

[6] E. Friedgut, "Hypergraphs, entropy and inequalities," The American Mathematical Monthly, vol. 111, no. 9, pp. 749-760, November 2004.

[7] R. G. Gallager, Low-Density Parity-Ceck Codes, MIT Press, Cambridge, MA, USA, 1963.

[8] D. Galvin, "Three tutorial lectures on entropy and counting," First Lake Michigan Workshop on Combinatorics and Graph Theory, March 2014. Available at https://arxiv.org/abs/1406.7872

[9] D. Galvin and Y. Zhao, "The number of independent sets in a graph with small maximum degree," Graphs and Combinatorics, vol. 27, no. 2, pp. 177-186, March 2011.

[10] D. Gavinsky, S. Lovett, M. Saks and S. Srinivasan, "A tail bound for read- $k$ families of functions," Random Structures and Algorithms, vol. 47, no. 1, pp. 99-108, August 2015.

[11] O. Johnson, I. Kontoyiannis and M. Mokshay, "Log-concavity, ultra-logconcavity, and maximum entropy property of discrete compound Poisson processes," Discrete Applied Mathematics, vol. 161, no. 9, pp. 1232 1250, June 2013.

[12] T. Kaced, A. Romashchenko and N. Vereshchagin, "A conditional information inequality and its combinatorial applications," IEEE Transactions on Information Theory, vol. 64, no. 5, pp. 3610-3615, May 2018.

[13] J. Kahn, "An entropy approach to the hard-core model on bipartite graphs," Combinatorics, Probability and Computing, vol. 10, no. 3, pp. 219-237, May 2001.

[14] J. Kahn, "Entropy, independent sets and antichains: a new approach to Dedekind's problem," Proceedings of the American Mathematical Society, vol. 130, no. 2, pp. 371-378, June 2001.

[15] M. Madiman and P. Tetali, "Sandwich bounds for joint entropy," Proceedings of the 2007 IEEE International Symposium on Information Theory, Nice, France, pp. 511-515, June 2007.

[16] M. Madiman and P. Tetali, "Information inequalities for joint distributions with interpretations and applications," IEEE Transactions on Information Theory, vol. 56, no. 6, pp. 2699-2713, June 2010.

[17] J. L. Massey, "On the fractional weight of distinct binary $n$-tuples," IEEE Transactions on Information Theory, vol. 20, no. 1, pp. 131-132, January 1974

[18] N. Pippenger, "An information-theoretic method in combinatorial theory," Journal of Combinatorial Theory, Series A, vol. 23, no. 1, pp. 99 104, July 1977.

[19] N. Pippenger, "Entropy and enumeration of boolean functions," IEEE Transactions on Information Theory, vol. 45, no. 6, pp. 2096-2100, September 1999.

[20] J. Radhakrishnan, "Entropy and counting," Proceedings of the IIT Kharagpur, Golden Jubilee Volume on Computational Mathematics, Modelling and Algorithms, Narosa Publishers, India, pp. 1-25, 2001.

[21] J. Radhakrishnan, "An entropy proof of Bregman's theorem," Journal of Combinatorial Theory, Series A, Elsevier Science, vol. 77, no. 1, pp. 161-164, January 1997.

[22] T. Richardson and R. Urbanke, Modern Coding Theory, Cambridge University Press, 2008.

[23] A. Sah, M. Sawhney, D. Stoner and Y. Zhao, "The number of independent sets in an irregular graph," Journal of Combinatorial Theory, Series B, vol. 138, pp. 172-195, September 2019.

[24] I. Sason, "A generalized information-theoretic approach for bounding the number of independent sets in bipartite graphs," Entropy, vol. 23, no. 3, paper 270, pp. 1-14, March 2021.

[25] V.G. Vizing, "On an estimate of the chromatic class of a $p$-graph," Diskret. Analiz., vol. 3, pp. 25-30, 1964.

[26] Y. Zhao, "The number of independent sets in a regular graph," Combinatorics, Probability and Computing, vol. 19, pp. 315-320, March 2010. 